\title{
Result Analysis and Comparison of Hybrid Method based on Local Binary Pattern (LBP) and Color Moment (CM) for Efficient Image Retrieval
}

\author{
Hardeep Singh \\ PhD Scholar \\ ECE Department \\ CPU Kota
}

\author{
Dheeraj Agrawal \\ Assistant Professor \\ ECE Department \\ MANIT Bhopal
}

\begin{abstract}
In this paper LBP and CM methods have been efficiently used for image retrieval for the content based image retrieval (CBIR) system. As LBP method may be sensible to noise in case of comparing neighboring pixels. The drawback of CM is it may be inefficient with too much details image. So it will be better to combine the feature of both method and utilized the property of both the two methods. In this paper LBP and CM are used combined along with the analysis of individual LBP and $\mathrm{CM}$ for the comparison. Wang database have been used for the experimentation. All the 10 classes are used for the results comparison. The results suggested that the combined method have the capability over individual methods in efficient image retrieval.
\end{abstract}

\section{Keywords}

CBIR, Content Retrieval, LBP and CM

\section{INTRODUCTION}

Visual analysis is an emerging trend in research arena. CBIR system is more trending now a days because of the demand of efficient image retrieval system [1]. The demand of efficient image retrieval system is the need of the current edge so that picture information is extracted properly [2-4]. Image information solidifies the noisy pictures and data secluded from pictures through electronic or recreated estimations [5].

CBIR systems are similarly working with the same lay [6]. A section vector is ousted from each photo in the database and the course of action of all highlight vectors is encircled as a database record [7]. At the time, a substitution vector is removed from the solicitation picture other than; it is energized against the section vectors in the record [8-16]. The key differentiation between the specific structures lies in the parts that they empty and in the counts that are utilized to take a gander at highlight vectors [17]. The shading is the most by and large used visual fragments as a piece of picture recuperation in light of the way that they are less requesting to withdraw isolated and surface and shape information. Shading highlight is acceptably ready to establishment multifaceted nature and free of picture size and presentation. Quantifiably, it demonstrates the joint probability of the intensities of the three shading channels [18]. Blends of timetables have been made for measuring strategy equivalence [19]. Most systems rely on upon in the wake of taking a gander at estimations of what are known as second-demand estimations enrolled from sales and set away pictures [20]. These timetables figure measures of picture surface, for instance, the level of refinement, coarseness, directionality and consistency [21]; or periodicity, directionality and intercession [22]. Elective system for surface examination for picture recuperation merges the utilization of Gabor channels [23] and fractals [24].

\section{RELATED WORKS}

In 2011, Jain et al. [25] suggested that the CBIR is a widely used procedure which is capable in finding images from huge image databases. With expansive information sets, there is probability of high dimensionality. In 2012, Das et al. [26] implemented a CBIR system which uses different feature of images by applying four methods, two methods were based on the color feature analysis and rest two were based on color and texture feature analysis using wavelet coefficients of an image. In 2007, Jain et al. [27] proposed an algorithm for retrieving images. The algorithm uses the shape information in an image along with its 3D information. The calculation utilizes the shape data as a part of a picture alongside its 3D data. In 2014, Wan et al. [28] suggested that the learning representations and similarity measures are very important for CBIR system. They have suggested that semantic gap issue which can be exists in the low-level image pixels which are only captured by machines and high-level semantic concepts perceived by human. In 2006, Heller et al. [29] presented a Bayesian framework for CBIR which is capable in modeling the distribution of color and texture features within sets of related images. They suggested that for a user specified content question the framework first concentrates an arrangement of images, from a named corpus, relating to that question. In 2012, Chaudhari et al. [30] suggested that the CBIR uses the visual properties of an image such as color, shape, texture, and spatial layout to represent and index the image. In 2015 Dubey et al. [31-34] suggested that data mining and evolutionary algorithms can be useful in improving the classification accuracy. So there used techniques may be helpful in image retrieval and proper classification. In 2007, Liu et al. [3] provided a comprehensive survey of the recent technical achievements in high-level semantic-based image retrieval. In 2015, Alzu'bi et al. [35] suggested that the multimedia contents complexity is increasing dominantly in the current digital world. So this generates the need of highly effective retrieval systems. In 2012, Jain et al. [36] presented an efficient framework with the combination of color, texture and shape information. They have applied the coordination of the above and bunch in view of alike properties. In view of five predominant colors they recover the comparative pictures. In 2013, Bhagat et al. [37] suggested that the execution of an electronic extensible design that is effortlessly indispensable with applications written in various dialects and linkable with various information sources. In [38] author has presented a brief survey on CBIR techniques. 


\section{METHODS}

In this paper LBP and CM methods are applied for the efficient image retrieval technique. It is a part of CBIR system which means content based image retrieval system. In this the data has been searched based on the content. Content indicates the colors, shapes and textures. LBP is used here as it is efficient in thresholding the neighborhood for every pixel. By this classification accuracy can be improved and it can help in feature selection [39]. CM is useful in indexing the images based on the color in image retrieval system as it provides image retrieval based on color index. For result validation Wang database have been used [40] [41]. It is a collection of 1000 database.

\section{LBP}

In this method of image retrieval first the color images are converted to gray scale. As it works only on gray scale images. As we have already discussed that it is efficient in neighborhood pixel selection and thresholding. Then LBP values for the corresponding pixels are calculated. Then corresponding pixel location is updated in the data matrix or used as the LBP mask vector. The neighboring pixel values are then compared with the central pixel value to calculate the pixel LBP value. It can be calculated for all the pixels. This can be done by traversing in the same order of clock or anticlock wise position with the same position. So the comparison is depends on the pixels. It is started from the initial pixel. These values are between 0 to 255 . The main benefit of using LBP is translation invariant property.

The whole image is considered for the process. LBP method is capable enough to extract the local information. It helps in extracting local features of an object. The main concept behind using the LBP technique is to calculate the local structure of an image by comparing the pixels with the neighborhood.

$\operatorname{LBP}\left(\mathrm{X}_{\mathrm{c}}, \mathrm{Yc}\right)=\sum_{n=0}^{n-1} 2^{n} \mathrm{Q}\left(\mathrm{I}_{\mathrm{n}}-\mathrm{I}_{\mathrm{c}}\right)$

Where $X_{c}$ and $Y_{c}$ denotes the central pixel. $Q$ can be understood as follows:

$\mathrm{Q}(\mathrm{X})=\left\{\begin{array}{rr}1 & \text { if } x \geq 0 \\ 0 & \text { else }\end{array}\right.$

$I_{n}$ denote the neighbor pixel intensity. $I_{c}$ denotes the central pixel intensity.

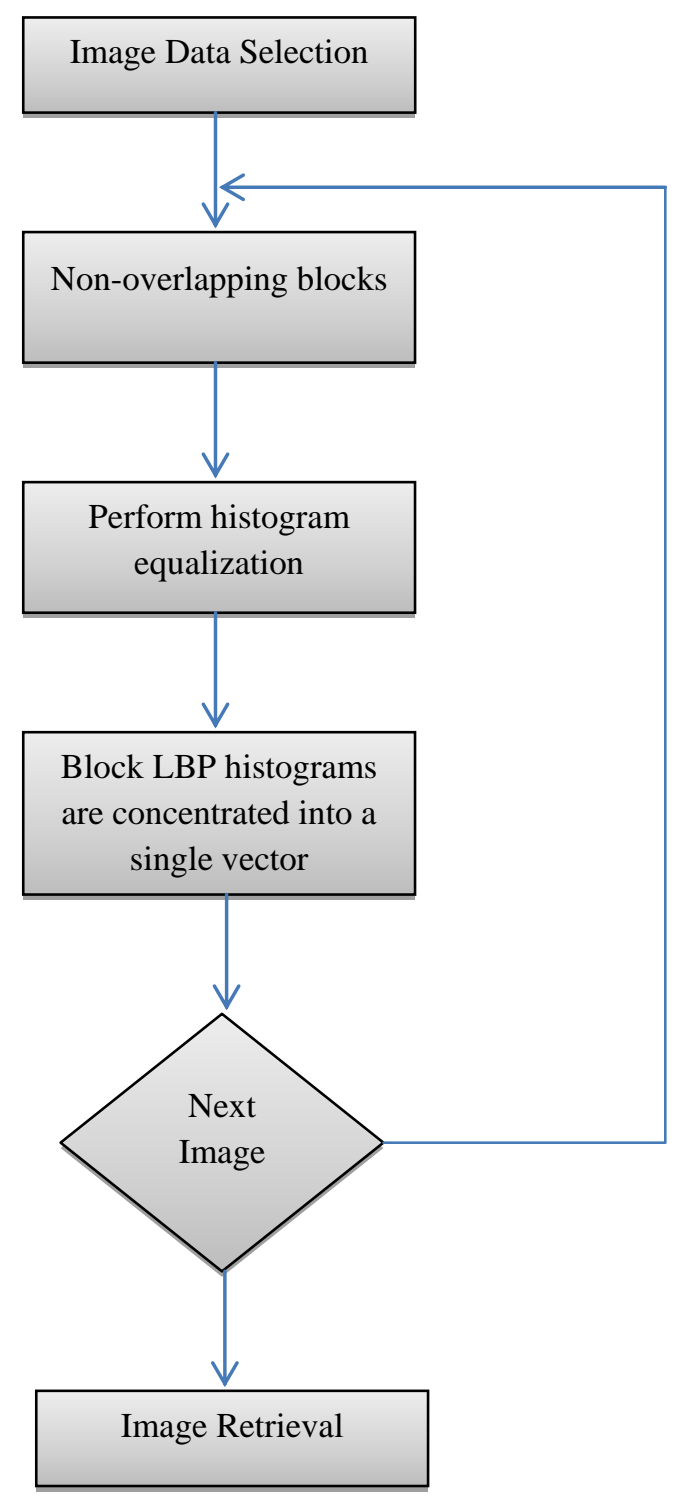

Figure 1: Process flowchart of LBP

For the calculated center vector $X_{c}$ and $Y_{c}$ the position of the neighbor in the LBP can be calculated as follows:

$X_{n}=X_{c}+R \cos \frac{2 \pi n}{S}$

$\mathrm{Y}_{\mathrm{n}}=\mathrm{Y}_{\mathrm{c}}-\mathrm{R} \sin \frac{2 \pi n}{S}$

Where $\mathrm{R}$ denotes radius and $\mathrm{S}$ is the sample point.

\section{CM}

It is a supportive measurement which can be used to differentiate images based on the color feature. It is calculated based on the similarity of images. This can be basically determined by the normal distribution or by calculating their mean and variance. It shows a pattern of moment which can help in identifying the image based on color. Three moments are used mainly mean, standard deviation and skewness. The color can be defined as the Hue, Saturation and brightness. Then the moments are calculated for the separate channel in the picture. Color moment can be formulated as follows:

Suppose there are n observations like $X_{1}, X_{2}, \ldots, X_{n}$ belong 
to $X$ variable. The average of $X_{1}, X_{2}, \ldots, X_{n}$ can be represented as is the mean, $\mu_{X}$, denoted as follows:

$\mu_{X}=\bar{X}=\frac{1}{n} \sum_{i=1}^{n} X_{i}$

Then the standard deviation can be calculated as follows:

$\sigma_{X}=\sqrt{\sigma_{X}^{2}}$. We have

$$
\sigma_{X}=\sqrt{\frac{1}{n}\left\{\sum_{i=1}^{n} X_{i}^{2}-\frac{1}{n}\left(\sum_{i=1}^{n} X_{i}\right)^{2}\right\}}
$$

According to the Karl skewness can be defined as follows:

$$
s k=\frac{\mu-\text { mode }}{\sigma}
$$

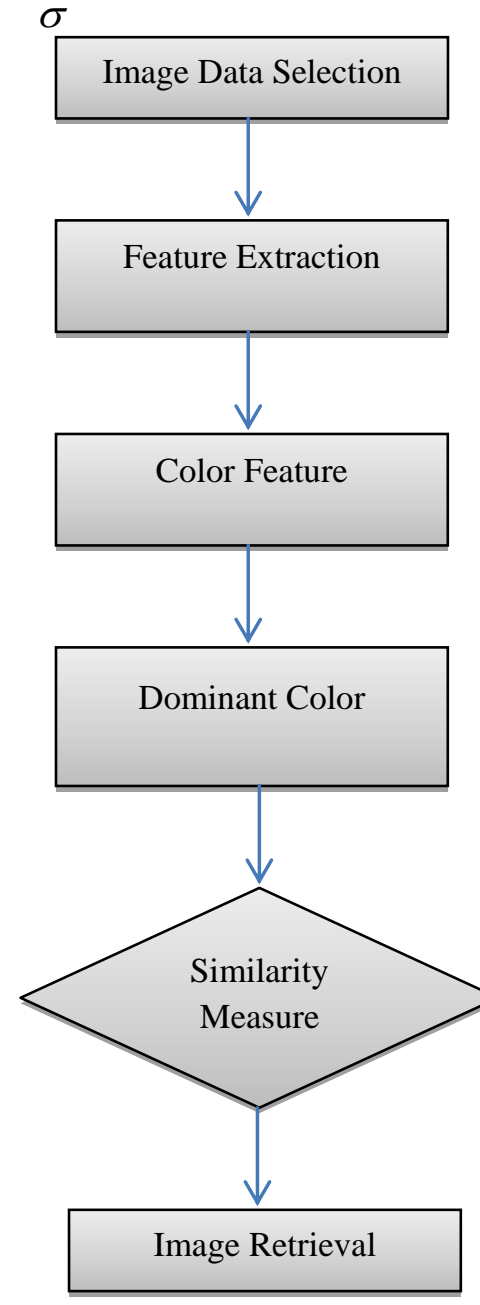

Figure 2: Process flowchart of CM

The group of color extracted based on the dominant color which is the combination of color fetched. Before separating the color attributes of a picture, all pixels on database pictures are arranged into comparable sorts of gatherings as per the similarity of their hues. A color will be chosen from predefined hues which are exceptionally close to picture pixel color and it is stored as a new pixel. Color distance $C_{D}$ can be measured by adopting Euclidean distance equation as specified below:
$C_{D}=\min \left(\sqrt{\left(R_{P}-R_{i T}\right)^{2}+\left(G_{P}-G_{i T}\right)+\left(B_{P}-B_{i T}\right)^{2}}\right)$

$R_{P}, G_{P}$ and $B_{P}$ are the color intensity values of red, green, and blue colors respectively. $R_{\mathrm{iT}}, \mathrm{G}_{\mathrm{iT}}$ and $\mathrm{B}_{\mathrm{iT}}$ are the color table index. The maximum percentage color component is selected as the dominant color and stored.

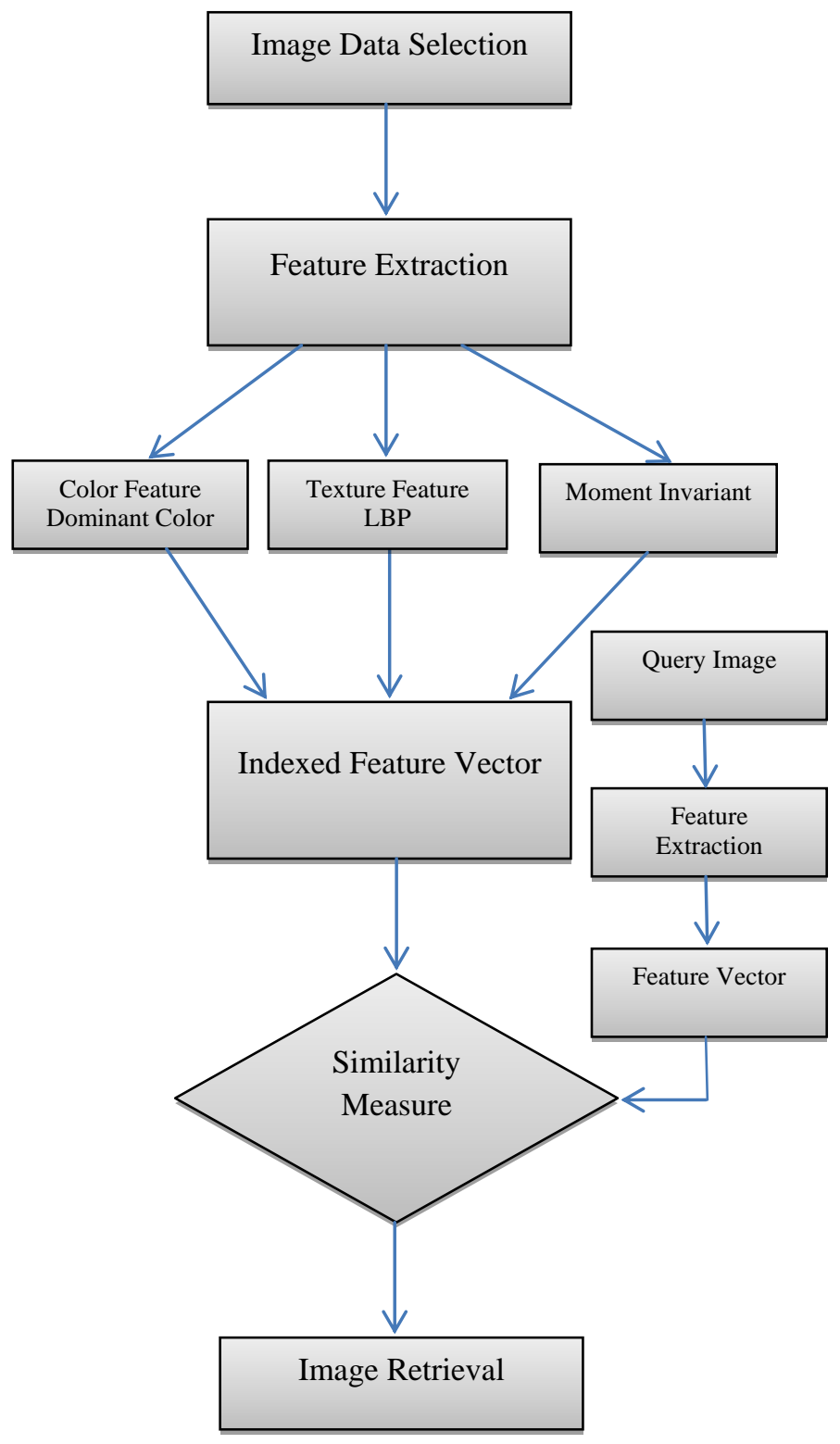

Figure 3: Combined flowchart for LBP and CM

For similarity measurement from the experimental images can be calculated as follows by the Euclidean distance, $\mathrm{d}$ using equation below:

$$
d=\sqrt{\left(F_{Q}[i]-F_{D B}[i]\right)^{2}}
$$

$F_{Q}[i]$ denotes $i^{\text {th }}$ feature of the query image and the $\mathrm{F}_{\mathrm{DB}}$ [i] indicates the related feature in the feature vector.

So it can be said that in color moment the images are first selected and moment for the query images are calculated then dominant color is used for the color feature extraction then similarity is calculated based on the rank. 
In this paper we have combined these two methodologies for achieving the better results.

\section{RESULTS AND DISCUSSION}

The results are compared based on the Wang database. All the ten classes are considered for experimentation. Figure 4 and figure 5 shows the process of LBP and CM. Different variations have been found in the results. It can suggest that the results are varying based on the properties of the images. Figure 6 shows the results based on the hybrid method and provide better results than individually applying LBP and $\mathrm{CM}$. Table 1 and figure 7 shows the overall comparison based on the LBP, CM and LBP + CM. It shows that the results outperforms in case of hybridization.

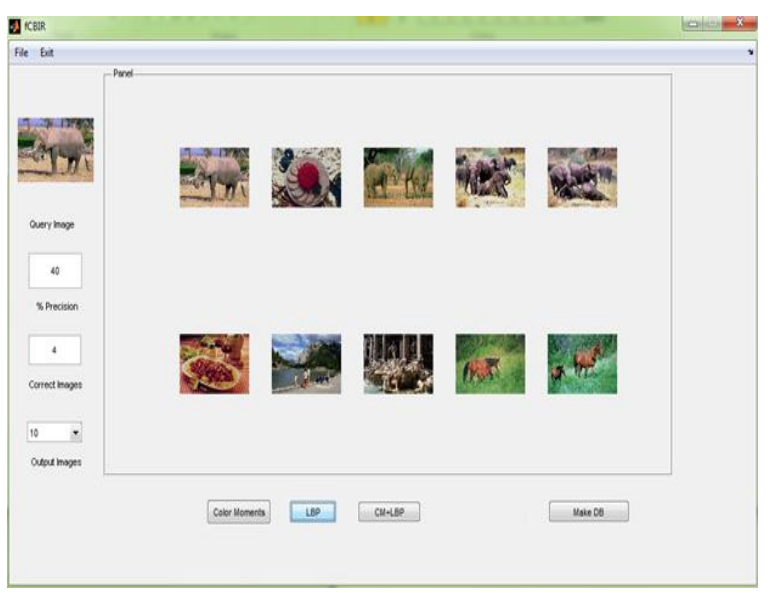

Figure 4: LBP for image database

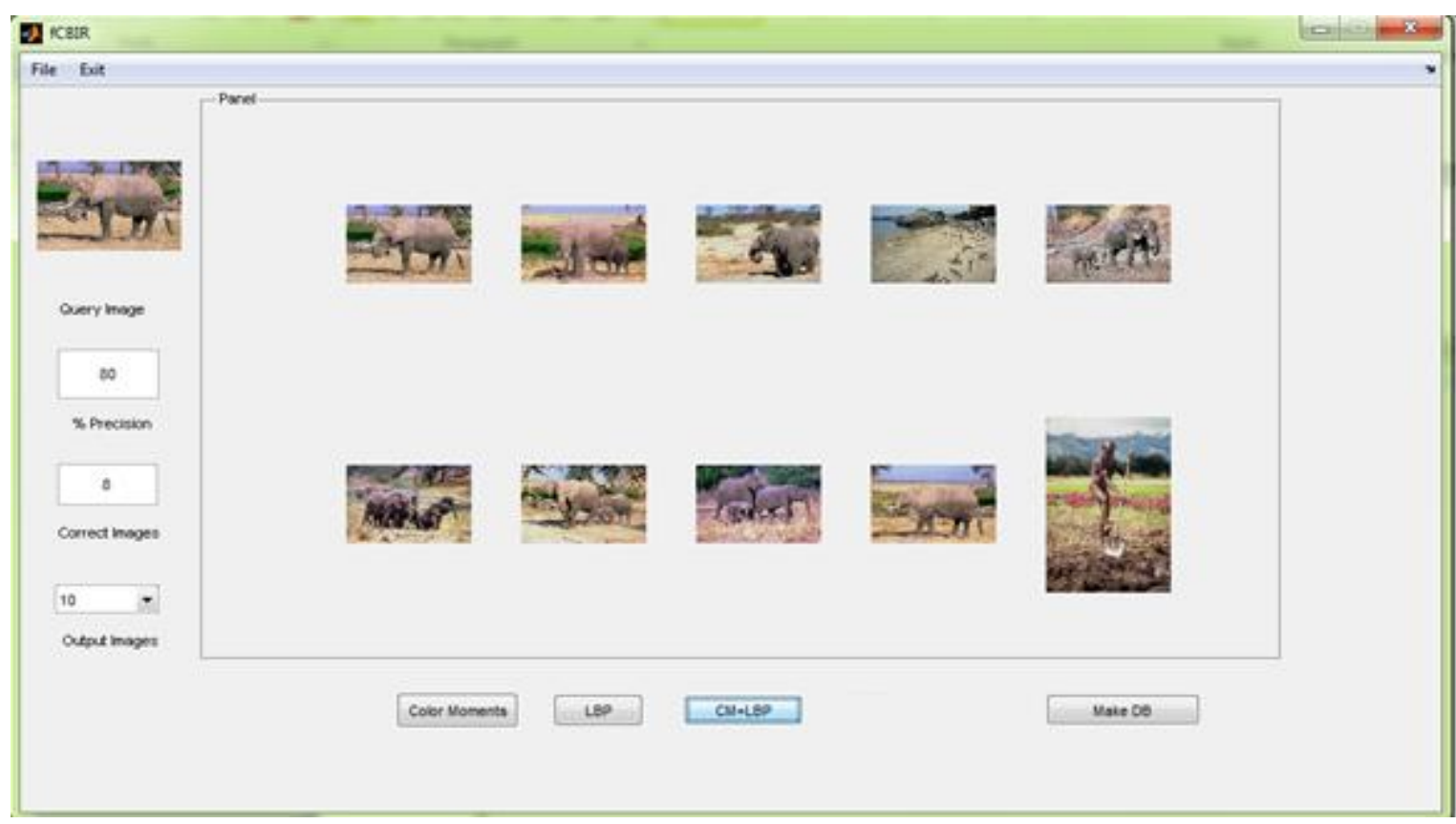

Figure 6: LBP + CM for image database

Table 1: Previous work comparisons

\begin{tabular}{|l|l|l|l|l|}
\hline S.no & Database Image & $\begin{array}{l}\text { Precision Method1- } \\
\text { LBP }(\%)\end{array}$ & $\begin{array}{l}\text { Precision Method2- } \\
\text { CM }(\%)\end{array}$ & $\begin{array}{l}\text { Precision Method3- } \\
\text { CM +LBP }(\%)\end{array}$ \\
\hline 1 & 1 & 80 & 80 & 80 \\
\hline 2 & 157 & 60 & 80 & 80 \\
\hline 3 & 219 & 10 & 30 & 30 \\
\hline
\end{tabular}




\begin{tabular}{|c|c|c|c|c|}
\hline 4 & 314 & 90 & 90 & 90 \\
\hline 5 & 400 & 100 & 100 & 100 \\
\hline 6 & 510 & 40 & 70 & 80 \\
\hline 7 & 640 & 90 & 70 & 70 \\
\hline 8 & 716 & 80 & 40 & 40 \\
\hline 9 & 814 & 30 & 80 & 60 \\
\hline 10 & 906 & 70 & 100 & 100 \\
\hline
\end{tabular}

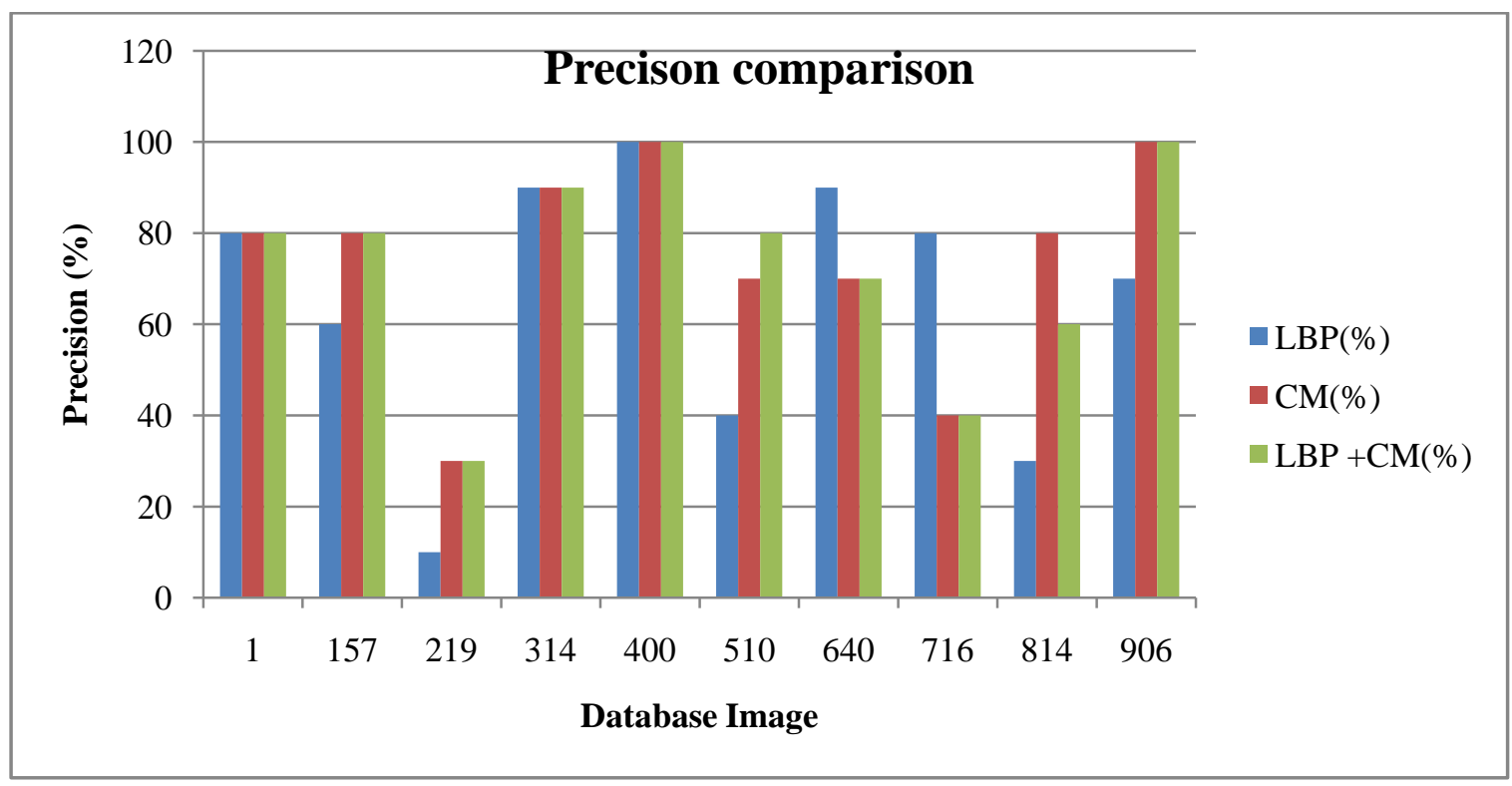

Figure 7: Comparative Table

\section{CONCLUSIONS}

In this paper LBP, CM and LBP + CM for the image retrieval based on CBIR system have been analyzed and discussed. The results have been discussed based on the LBP and CM. But the results obtained are variant and supports different combinations. But in the case of hybridization of LBP + CM better results have been obtained in comparison to using it individually.

\section{REFERENCES}

[1] Fu YQ, Wang YS. An algorithm for edge detection of gray-scale image based on mathematical morphology [J]. Journal of Harbin Engineering University. 2005; 5:027.

[2] Buades A, Coll B, Morel JM. A review of image denoising algorithms, with a new one. Multiscale Modeling \& Simulation. 2005; 4(2):490-530.

[3] Liu Y, Zhang D, Lu G, Ma WY. A survey of contentbased image retrieval with high-level semantics. Pattern recognition. 2007 Jan 31; 40(1):262-82.

[4] Prasanna MK, Rai SC. Image Processing Algorithms-A Comprehensive Study. International Journal of Advanced Computer Research. 2014 Jun 1; 4(2):532.

[5] Anandan P, Sabeenian RS. Curvelet based Image Compression using Support Vector Machine and Core Vector Machine-A Review. International Journal of Advanced Computer Research. 2014 Jun 1; 4(2):673.

[6] Kato T. Database architecture for content-based image retrieval. InSPIE/IS\&T 1992 symposium on electronic imaging: science and technology 1992 Apr 1 (pp. 112123). International Society for Optics and Photonics.
[7] Flickner M, Sawhney H, Niblack W, Ashley J, Huang Q, Dom B, Gorkani M, Hafner J, Lee D, Petkovic D, Steele D. Query by image and video content: The QBIC system. Computer. 1995 Sep; 28(9):23-32.

[8] Corea F. Emotional speculative behavior in the option market. International Journal of Advanced Computer Research. 2016 Jan 1; 6(22):18.

[9] Pentland A, Picard RW, Sclaroff S. Photobook: Contentbased manipulation of image databases. International journal of computer vision. 1996 Jun 1; 18(3):233-54.

[10] Smith JR, Chang SF. VisualSEEk: a fully automated content-based image query system. In Proceedings of the fourth ACM international conference on Multimedia 1997 Feb 1 (pp. 87-98). ACM.

[11] Wang JZ, Wiederhold G, Firschein O, Wei SX. Contentbased image indexing and searching using Daubechies' wavelets. International Journal on Digital Libraries. 1998 Mar 1;1(4):311-28.

[12] Carson C, Belongie S, Greenspan H, Malik J. Blobworld: Image segmentation using expectation-maximization and its application to image querying. IEEE Transactions on Pattern Analysis and Machine Intelligence. 2002 Aug; 24(8):1026-38.

[13] Wang JZ, Li J, Wiederhold G. SIMPLIcity: Semanticssensitive integrated matching for picture libraries. IEEE Transactions on pattern analysis and machine intelligence. $2001 \mathrm{Sep} ; 23(9): 947-63$. 
[14] Puviarasan N, Bhavani R. Retrieval of Images Using Weighted Features. International Journal of Advanced Computer Research. 2014 Mar 1; 4(1):60.

[15] Patidar D, Jain N, Nagariya B, Mishra M. Image Classification by Combining Wavelet Transform and Neural Network. International Journal of Advanced Computer Research. 2013 Dec 1; 3(4):106.

[16] Singh H, Kaur T. Novel Method for Edge Detection for Gray Scale Images using $\mathrm{VC}++$ Environment. International Journal of Advanced Computer Research. 2013 Dec 1; 3(4):193.

[17] Mathur A, Mathur R. Content Based Image Retrieval by Multi Features using Image Blocks. International Journal of Advanced Computer Research. 2013 Dec 1; 3(4):251.

[18] Viswa SS. Efficient retrieval of images for search engine by visual similarity and re ranking. International Journal of Advanced Computer Research. 2013 Jun 1; 3(2):47.

[19] Eakins JP, Graham ME. Content based image retrieval: A report to the JISC technology applications programme. Cyberabstracts.

[20] Tamura H, Mori S, Yamawaki T. Textural features corresponding to visual perception. IEEE Transactions on Systems, Man, and Cybernetics. 1978 Jun; 8(6):46073.

[21] Zhang S, Bao CW, Shen HB. Independent component analysis based on adaptive artificial bee colony. International Journal of Advanced Computer Research. 2016; 6(25):146-152.

[22] Liu F, Picard RW. Periodicity, directionality, and randomness: Wold features for image modeling and retrieval. IEEE transactions on pattern analysis and machine intelligence. $1996 \mathrm{Jul} ; 18(7): 722-33$.

[23] Kaplan LM, Murenzi R, Namuduri KR. Fast texture database retrieval using extended fractal features. InPhotonics West'98 Electronic Imaging 1997 Dec 23 (pp. 162-173). International Society for Optics and Photonics.

[24] Smith JR. Integrated spatial and feature image system: Retrieval, analysis and compression [Ph D dissertation]. Columbia University, New York. 1997.

[25] Jain M, Singh SK. A survey on: content based image retrieval systems using clustering techniques for large data sets. International Journal of Managing Information Technology. 2011 Nov 1;3(4):23.

[26] Das S, Garg S, Sahoo G. Comparison of Content Based Image Retrieval Systems Using Wavelet and Curvelet Transform. The International Journal of Multimedia \& Its Applications. 2012 Aug 1; 4(4):137.

[27] Jain A, Muthuganapathy R, Ramani K. Content-based image retrieval using shape and depth from an engineering database. In International Symposium on Visual Computing 2007 Nov 26 (pp. 255-264). Springer Berlin Heidelberg.

[28] Wan J, Wang D, Hoi SC, Wu P, Zhu J, Zhang Y, Li J. Deep learning for content-based image retrieval: A comprehensive study. In Proceedings of the 22nd ACM international conference on Multimedia 2014 Nov 3 (pp. 157-166). ACM.
[29] Heller KA, Ghahramani Z. A simple Bayesian framework for content-based image retrieval. In2006 IEEE Computer Society Conference on Computer Vision and Pattern Recognition (CVPR'06) 2006 (Vol. 2, pp. 2110-2117). IEEE.

[30] Chaudhari R, Patil AM. Content based image retrieval using color and shape features. International Journal of Advanced Research in Electrical, Electronics and Instrumentation Engineering. 2012 Nov; 1(5).

[31] Dubey AK, Gupta U, Jain S. Breast cancer statistics and prediction methodology: a systematic review and analysis. Asian Pacific journal of cancer prevention: APJCP. 2014 Dec; 16(10):4237-45.

[32] Dubey AK, Gupta U, Jain S. A Survey on Breast Cancer Scenario and Prediction Strategy. InProceedings of the 3rd International Conference on Frontiers of Intelligent Computing: Theory and Applications (FICTA) 2014 2015 (pp. 367-375). Springer International Publishing.

[33] Dubey AK, Gupta U, Jain S. Epidemiology of lung cancer and approaches for its prediction: a systematic review and analysis. Chinese Journal of Cancer. $2016 \mathrm{Jul}$ $30 ; 35(1): 1$.

[34] Dubey AK, Gupta U, Jain S. Analysis of k-means clustering approach on the breast cancer Wisconsin dataset. International Journal of Computer Assisted Radiology and Surgery. 2016 Jun 16:1-5.

[35] Alzu'bi A, Amira A, Ramzan N. Semantic content-based image retrieval: A comprehensive study. Journal of Visual Communication and Image Representation. 2015 Oct $31 ; 32: 20-54$.

[36] Jain N, Sharma S, Sairam RM. Result Analysis on Content Base Image Retrieval using Combination of Color, Shape and Texture Features. International Journal of Advanced Computer Research (IJACR). 2012;2.

[37] Bhagat AP, Atique M. Web based image retrieval system using color, texture and shape analysis: comparative analysis. International Journal of Advanced Computer Research. 2013 Sep 1; 3(3):58.

[38] Dubey A. Efficient Content based Image Retrieval (CBIR) Techniques: A Survey. ACCENTS Transactions on Image Processing and Computer Vision (TIPCV). $2015 ; 1(1): 28-32$

[39] Kumar E, Khan Z, Jain A. A review of content based image classification using machine learning approach. International Journal of Advanced Computer Research 2012; 2(5):55-60

[40] Li J, Wang JZ. Automatic linguistic indexing of pictures by a statistical modeling approach. IEEE Transactions on pattern analysis and machine intelligence. 2003 Sep; 25(9):1075-88

[41] Wang JZ, Li J, Wiederhold G. SIMPLIcity: Semanticssensitive integrated matching for picture libraries. IEEE Transactions on pattern analysis and machine intelligence. $2001 \mathrm{Sep}$; 23(9):947-63 